\title{
ADICCIÓN A REDES SOCIALES E INTELIGENCIA EMOCIONAL EN ESTUDIANTES DE EDUCACIÓN SUPERIOR TÉCNICA
}

ADDICTION TO SOCIAL NETWORKS AND EMOTIONAL INTELLIGENCE IN TECHNICAL HIGHER EDUCATION STUDENTS

\author{
Giovanni Franklin Alarcón-Allaín \\ Universidad Peruana de Ciencias Aplicadas \\ https://orcid.org/0000-0002-6394-3300 \\ giovannialarconallain@gmail.com
}

Edwin Salas-Blas

Universidad Peruana de Ciencias Aplicadas, Universidad de San Martín de Porres https://orcid.org/0000-0002-0625-0313 e.salasb@hotmail.com

Correspondencia:

La correspondencia referida a este artículo debe dirigirse a Edwin Salas-Blas

Cómo citar este texto:

Alarcón-Allaín, G.F. y Salas-Blas, E. (2022). Adicción a Redes Sociales e Inteligencia Emocional en estudiantes de educación superior técnica. Health and Addictions / Salud y Drogas, 22(1), 152-166. doi: 10.21134/haaj.v22i1.640 


\section{Resumen}

Introducción. Las redes sociales son usadas por alrededor de 3,600 millones de personas, ellas facilitan la comunicación y favorecen los vínculos humanos; pero su uso inadecuado, podría ocasionar problemas y en el extremo adicción. Objetivo: Relacionar adicción a redes sociales e inteligencia emocional. Método: Estudio correlacional, participaron 279 estudiantes de nivel técnico-superior; se administraron el Cuestionario de Adicción a Redes Sociales (ARS) y la Escala de Inteligencia Emocional (IE) de Wong-Law. Resultados: Obsesión (ARS) correlaciona negativamente con Uso de las propias emociones y Regulación de las emociones; igualmente, Falta de control y Uso excesivo (ARS) con las dimensiones Valoración de las propias emociones, Uso de las propias emociones y Regulación de las emociones; pero, ningún factor del ARS correlaciona con Valoración de las Emociones de los demás; asimismo, hombres y mujeres no se diferencian en la ARS, los que tienen más horas de conexión son más propensos a mostrar puntajes más altos en adicción a redes; y, en cuanto a la inteligencia emocional, las mujeres valoran más las emociones de los demás. Conclusiones: La adicción a las redes sociales y la inteligencia emocional se relacionan negativamente en casi todas sus dimensiones, pero es una asociación de baja magnitud.

\section{Abstract}

Introduction. Social networks are used by around 3,600 million people, they facilitate communication and promote human ties; but its inappropriate use could cause problems and extreme addiction. Objective: Relate addiction to social networks and emotional intelligence. Method: Correlational study, 279 students of technical-superior level participated; The Social Media Addiction Questionnaire (ARS) and the Wong-Law Emotional Intelligence Scale (EI) were administered. Results: Obsession (ARS) negatively correlates with Use of one's own emotions and Regulation of emotions; likewise, Lack of control and Excessive use (ARS) with the dimensions Valuation of one's own emotions, Use of one's own emotions and Regulation of emotions; but, no factor of the ARS correlates with Valuation of the Emotions of the others; Likewise, men and women do not differ in the ARS, those who have more hours of connection are more likely to show higher scores in network addiction; and, in terms of emotional intelligence, women value the emotions of others more. Conclusions: Addiction to social networks and emotional intelligence are negatively related in almost all their dimensions, but it is an association of low magnitudes.

\section{Palabras clave}

Adicciones comportamentales, adicción a internet, adicción a redes sociales, inteligencia emocional, regulación de las emociones.

\section{Keywords}

Behavioral addictions, internet addiction, addiction to social networks, emotional intelligence, emotion regulation. 


\section{Introducción}

En el mundo existían hasta antes de la pandemia, más de 4,570 millones de usuarios activos de internet; de ellos, 3,600 millones usaban Redes Sociales (RS); el noreste de Asia y Norteamérica son las zonas de mayor penetración de las RS con $70 \%$ y $61 \%$ respectivamente (Clement, 2020). En Latinoamérica la penetración de internet sobrepasa también el 70 \% (Matassi \& Boczkowski, 2020); en Perú, según el Instituto Nacional de Estadística e Informática [INEI], el 78.1\% de personas utiliza internet a diario, de este, el 89,8\% lo usa para comunicarse por correo, chat o algunas de las RS (INEI, 2020).

Algunos estudios sobre la prevalencia de adicción a internet, reportan que en adolescentes japoneses es de $21.7 \%$ (Kawabe, Horiuchi, Ochi, Oka \& Ueno, 2016); en China, fluctúa entre los 22,5\% y 26,7\% (Yu \& Shek, 2013); A nivel latinoamericano, se encontró un $7.6 \%$ de personas con alto riesgo de adicción a las redes sociales (Fuster, Chamarro \& Oberst, 2017) y en adolescentes peruanos, un 16 \% de prevalencia (Pomalima et al., 2016).

El uso de internet y de RS tiene varias motivaciones: negocios, trabajo, estudios, relaciones amicales, información en general y diversión; en ella, los usuarios pueden disponer de su propia página online, manejar a voluntad sus perfiles, colgar fotos, comentar su propia experiencia de vida o la de otros y compartir información (Chóliz \& Marco, 2012). El uso del Internet, relacionado a las RS se ha incrementado debido a la mayor capacidad de conexión que tiene la población, a la tecnología cuyo avance lo facilita y a las características de los adolescentes y jóvenes que son los principales usuarios. Las RS permiten que las personas se conecten en tiempo real, independientemente de las distancias (Hütt, 2012), por lo que, estos aplicativos se han hecho indispensables para la comunicación sobre todo de las nuevas generaciones.

Si bien se puede encontrar aspectos positivos y benéficos en el uso de internet y de las RS; el uso excesivo, inadecuado, irresponsable y malicioso puede generar un sinnúmero de riesgos para los usuarios, especialmente para los adolescentes y jóvenes (Andreassen, 2015; Matute, 2016; Salas, 2014; Salas-Blas, 2019a, 2019b; Salas-Blas et al., 2020; Turel \& Serenko, 2012).

Definir la adicción a RS no es fácil, dentro de la literatura sobre el tema, se encuentran algunos términos que se usan sin precisar adecuadamente sus alcances y con significados confusos, como el uso excesivo, uso abusivo, uso problemático, uso patológico, adicciones y dependencias (Carbonell et al., 2018; Carbonell \& Oberst, 2015; García del Castillo et al., 2019; Matute, 2016; Salas-Blas et al., 2020; Turel \& Serenko, 2012). El uso excesivo tiene que ver con el tiempo o la frecuencia de uso, que puede estar motivado por cuestiones laborales o de estudio, puede ser una cuestión temporal, un fenómeno relacionado con los atractivos y el reforzamiento positivo que el usuario recibe, por la novedad de su descubrimiento, etc.; pero, el uso excesivo no constituye necesariamente una adicción (Matute, 2016; Turel \& Serenko, 2012); otro término similar es el de abuso de internet que también se desestima como adicción, porque carece de las características de una adicción y se relaciona con el uso excesivo (Carbonell \& Oberst, 2015; Carbonell et al., 2018). Matute (2016) plantea otro concepto, el de uso problemático, para referirse a los casos de uso desregulado, impulsivo y descontrolado, que generan algunas dificultades en las relaciones con los demás, que puede crear patrones cognitivos y conductuales con apariencia de dependencia; considera que éste concepto integra algunas conductas que pueden relacionarse con problemas de salud, de integración social y familiar, con problemas académicos o de trabajo, pero que tampoco constituye un problema de adicción. Las personas con exceso de uso y que hacen un uso problemático de las RS pueden pasar largos periodos de tiempo conectados, pero no necesariamente ser adictos; aunque algunos casos de estos, podrían considerarse como tales, siempre y cuando, desarrollen características de abstinencia, tolerancia, aislamiento, depresión y ansiedad, similares a los que se encuentran en las adicciones a sustancias (Andreassen, 2015; Chóliz \& Marco, 2012; Echeburúa \& Requesens, 2012; Escurra \& Salas, 2014; Matute, 2016).

Para algunos autores la dependencia a internet y a las RS sigue ciertos patrones en su formación, empieza con un exceso de uso, en algunos casos abuso, que se convierte en un hábito (Turel \& Serenko, 2012); seguida por la pérdida de la capacidad para controlar su uso, y, luego del desarrollo de los síntomas de tolerancia y abstinencia (Caro, 2017) que caracterizan a la adición; pero, no existen estudios concluyentes sobre el potencial poder adictivo de las 
RS (Carbonell \& Oberst, 2015; Caro, 2017; Echeburúa, 2016; Matute, 2016). Algunos autores sostienen que existe un perfil de personas propensas a sufrir adicciones (Echeburúa \& De Corral, 2010), que este fenómeno puede generar un incremento de los neurotransmisores relacionados al placer (Echeburúa \& Requesens, 2012; Chóliz \& Marco, 2012); los mismos que serían la base para el desarrollo de las características esenciales de la adicción como la abstinencia, la tolerancia y una valoración extrema de estímulos emocionales. En cuanto se puede hablar de adicción, los problemas con los que se relaciona pueden clasificarse en cuatro tipos: de relaciones con los demás, emocionales, problemas de salud y de rendimiento (Aguilar et al., 2008; Andreassen, 2015; Granados et al., 2020).

Una definición de adicción a redes sociales, podría considerar una excesiva preocupación e impulsos incontrolados por acceder y permanecer en las redes sociales, un aislamiento de su entorno habitual y ciertos trastornos de tipo cognitivo y emocional (Andreassen \& Pallesen, 2014; Escurra \& Salas, 2014; Granados et al., 2020). La personalidad, autoestima, vulnerabilidad psicológica, ansiedad, así como el estrés podrían ser factores de riesgo para desarrollar una adicción a nuevas tecnologías como las RS; asimismo, los factores de protección estarían relacionados con las habilidades de afrontamiento, el entorno social sano y el apoyo familiar constante (Echeburúa, 2012; Andreassen \& Pallesen, 2014; Young et al., 2019).

Asimismo, se postula que las RS influyen en la construcción de la identidad de los adolescentes y jóvenes (Arab \& Díaz, 2015), ellas son reguladoras de sus estados de ánimo, sobre todo de sus sentimientos de soledad y de las situaciones de estrés (Leung, 2007). Por otro lado, Watters et al. (2013) mencionan que cuando una persona se siente incompetente a nivel social, tiene mayor preferencia por la conexión en línea; probablemente, es más sencillo estar conectado que estar relacionado, porque, en las redes sociales no se necesita utilizar las habilidades blandas e implica poco esfuerzo para entablar vínculos persona-persona (Bauman, 2011).

Salovey \& Mayer (1990), Goleman (1995) y otros, postulan que la inteligencia emocional [IE] tiene que ver con el uso adaptativo de nuestras propias emociones en favor de un razonamiento más efectivo. Por ende, la IE sería la interrelación entre competencias no cognitivas y habilidades que influyen en el afrontamiento de situaciones de forma exitosa (Bar-On, 1997). Ella podría considerarse también como la habilidad que tienen los individuos para lidiar con las emociones propias como extrañas e incluyen cuatro áreas: Valoración y expresión de emociones propias, valoración y reconocimiento de las emociones del otro, regulación de las propias emociones y el uso de las emociones para facilitar el desempeño (Law et al., 2004; Wong \& Law, 2002). Asimismo, Para (Goleman, 2011), la inteligencia emocional es la habilidad de persistir ante frustraciones, controlar impulsos y demorar las recompensas

La regulación de las emociones puede favorecer a la mejora de algunos problemas psicopatológicos y conductas problemáticas, entre ellas la depresión, los trastornos alimenticios, el consumo de sustancias adictivas (Marino et al., 2019; Sloan et al., 2017). A partir de estos conocimientos se podría postular que si se fortalece la inteligencia emocional podría mantenerse el equilibrio entre el uso de redes sociales y la dependencia a ellas. Sin embargo, el problema radica en el poder de atracción que posee el internet, en la facilidad para su acceso y su costo cada vez menor; igual, la intimidad, el anonimato y la alta estimulación; que podrían conducir al desarrollo de conductas de uso problemático de las RS y en algunos casos de adicciones (Echeburúa, 2012; Matute, 2016).

Diversos autores consideran que los adolescentes entre 17 y 19 años son los más propensos a abusar del internet (Arthanari et al., 2017; Gómez et al., 2017; Perrella \& Caviglia, 2017), este es un rango etario denominado de los nativos digitales (IPSOS, 2018), que estarían más predispuestos a desarrollar adicciones; se sugiere también, que esta predisposición es mayor en jóvenes de ciudades que poseen fácil acceso y conexión a internet (Pomalima et al., 2016; Matalinares et al., 2013); del mismo modo, algunos sitios web contienen múltiples situaciones de juegos, de expresiones emocionales de la ira, miedo, sorpresa (Peña \& Rubio, 2013) en el que participan adolescentes y jóvenes, quienes serían especialmente sensibles al uso excesivo, problemático o adictivo de las RS (García del Castillo et al. 2019; Plaza, 2016).

Estudios antecedentes sugieren la existencia de correlación entre adicción a redes sociales y la inteligencia emocional (Bastidas, 2020; Contreras \& Curo, 2017; Jaramillo 2018; Mizera et al., 2019), se han encontrado correlaciones inversas bajas y muy bajas entre los dos constructos y algunas de sus dimensiones. Pero existen también estudios que utilizaron variables semejantes y que se acercan a lo que teóricamente se prevé (Granados et al., 2020; Hormes 
et al., 2014; Pontes et al., 2018). Khoshakhlagh \& Faramarzi (2012) encontraron correlaciones estadísticamente significativas entre adicción a internet, inteligencia emocional y trastornos mentales. Igualmente, hay evidencias de que algunas adicciones comportamentales como el juego patológico, la compra compulsiva o la adicción al sexo están relacionadas con factores emocionales y la necesidad de aliviar el malestar de forma inmediata (Cía, 2017; Echeburúa et al., 2014; Sola et al., 2013).

Los resultados del presente estudio tienen importancia teórica, puesto que la relación entre adicciones conductuales e inteligencia emocional, no está firmemente establecida empíricamente, pero también podría tener importancia aplicada para que más adelante se puedan desarrollar acciones preventivas orientadas a jóvenes con riesgo adictivo. Además, se espera que la relación de las variables investigadas quede como base para que futuras investigaciones puedan replicarlas en otras poblacionales.

El objetivo principal es relacionar ARS e IE entre estudiantes de educación técnica superior. Asimismo, hacer un análisis de coeficiente de determinación considerando la IE como la variable independiente y ARS como la variable dependiente. Y comparar la ARS y IE sobre la base del sexo y la frecuencia de conexión a redes sociales por día; comparar también la inteligencia emocional teniendo en cuenta puntuaciones altas y bajas del ARS

\section{Método}

El presente estudio se desarrolla con una estrategia asociativa y un diseño correlacional (Ato et al., 2013).

\section{Participantes}

Se trabajó con 279 jóvenes voluntarios de 18 a 23 años, 144 mujeres, estudiantes de distintas carreras técnicas de un instituto estatal del Callao-Perú; es una muestra no probabilística, elegida por su disponibilidad (Del Cid, Méndez \& Sandoval, 2011). El tamaño de la muestra se calculó con el programa G*Power (versión 3.1.9.2) (Faul et al., 2007), considerando el valor del coeficiente de correlación reportado en el estudio de Saraiva et al. (2018), que determinó un mínimo de 266 personas. Los criterios de inclusión consideraron la edad, que usen por lo menos una red social, y, a estudiantes que no hayan repetido el semestre; se excluyó a jóvenes influencers o a los que trabajan con redes sociales. Se escogió este rango de edades por el hecho de ser considerados nativos digitales (IPSOS, 2018).

\section{Instrumentos}

La ficha sociodemográfica fue construida específicamente para este estudio, explora algunos datos generales de los participantes: sexo, edad, carrera elegida, ciclo de estudios, frecuencia con que se conecta a redes sociales, etc.

El cuestionario de Adicción a Redes Sociales (ARS), construido por Escurra y Salas (2014) está compuesta por tres factores: obsesión por las redes sociales, con 10 ítems; falta de control personal en el uso de las redes sociales, con seis ítems; $y$, uso excesivo de las redes sociales, con ocho ítems. Los autores utilizaron el análisis factorial confirmatorio, con adecuados índices de ajustes en los tres factores: $(\chi 2(238)=35.23 ; p<.05 ; \chi 2 / g /=1.48 ; \mathrm{GFI}=.92 ; \mathrm{RMR}$ $=.06 ; \mathrm{RMSEA}=.04 ; \mathrm{AIC}=477.28)$, que brindan evidencias de validez factorial adecuada. La confiabilidad reportada por los autores, arroja coeficientes alfa de Cronbach mayores a .88 (altos) en los tres factores (DeVellis, 2011); las preguntas tienen una escala de respuestas tipo Likert del 0 (siempre) al 4 (nunca). En la presente investigación se estimó la consistencia interna mediante el coeficiente omega ( $\omega$, Ventura-León \& Caycho, 2017), reportándose que la dimensión Obsesión por las redes sociales presenta $\boldsymbol{\omega}=.76$, Uso excesivo de las redes sociales $\boldsymbol{\omega}=.77$ y Falta de control personal en el uso de redes sociales $\omega=.58$, valores medios, pero aceptables (Katz, 2011).

Escala de Inteligencia Emocional de Wong-Law (WLEIS) desarrollada por Wong y Law (2002). Presenta 16 ítems, distribuidos en 4 dimensiones: Valoración de las propias emociones ( $\alpha .87)$, Valoración de emociones de otros ( $\alpha .90)$, Uso de propias emociones ( $\alpha .84$ ) y Regulación de emociones ( $\alpha .83$ ); utiliza un formato de respuesta con una escala 
tipo Likert de 7 puntos (1 totalmente en desacuerdo a 7 totalmente de acuerdo). En el Perú fue validado por Merino et al. (2016)creada para el contexto laboral y que evalúa valoración y expresión de las emociones propias, valoración y reconocimiento de las emociones en otros, regulación de las propias emociones y uso de la emoción para facilitar el desempeño. Hay poca evidencia psicométrica sobre el Wong-Law Emotional Intelligence Scale en habla hispana, lo que posiblemente conduce a que sea también poco utilizado en la investigación y práctica profesional. El objetivo del presente estudio es presentar los primeros resultados de la validez de la estructura interna del WLEIS en adultos peruanos. Fueron 120 participantes (72 mujeres, con una muestra de 120 personas entre los 17 y 59 años; con respecto a la confiabilidad estimada por el coeficiente $\alpha$. Todas sus magnitudes obtuvieron resultados superiores a .85 , lo que indica una adecuada confiabilidad de la prueba. Asimismo, en el presente estudio se calculó la confiabilidad mediante el coeficiente omega $(\omega)$, obteniéndose valores satisfactorios (Katz, 2011) en todas las sub escalas: Valoración de las propias emociones $(\omega=.79)$, Valoración de emociones de otros $(\omega=.72)$, Uso de propias emociones $(\omega=.81)$ y Regulación de emociones $(\omega=.86)$.

\section{Procedimiento}

Los autores de las pruebas concedieron permiso para utilizar los instrumentos; asimismo, a través de la UPC se solicitó permiso para aplicarlos en la institución educativa elegida, y se coordinó las fechas de la administración en las propias aulas de clase, los ambientes fueron adecuados para los propósitos del estudio.

La aplicación de las escalas fue colectiva, participaron los estudiantes que cumplían con los criterios de inclusión mencionados. Antes de la aplicación, se les explicó los objetivos del estudio y que su participación era voluntaria, que se garantizaba el anonimato y que los datos recolectados serían de carácter estrictamente confidencial y sólo para ser usados en el estudio. Luego se les entregó un formato de consentimiento informado y se les leyó las instrucciones de cada una de las escalas, que además estaban escritas en la en la parte superior de las mismas.

Se les indicó que en las hojas de respuesta sólo podían marcar una respuesta por ítem, la marca la realizaron con lapicero, la administración en conjunto duró aproximadamente 25 minutos. Finalmente, se eliminaron los cuestionarios que tenían datos incompletos o que respondieron inadecuadamente; los datos recolectados se digitalizaron en Excel.

El análisis de los datos se efectuó con el programa estadístico Jamovi 1.0.7.0 (The Jamovi project, 2019). El estadístico de contraste Kolmogórov-Smirnov, reveló que los datos no se aproximaban a una distribución normal, por lo que se decidió utilizar el coeficiente de correlación de Spearman y para las comparaciones la prueba U de Mann-Whitney, además se calculó el tamaño del efecto.

\section{Resultados}

La tabla 1 reporta que algunos factores del ARS presentan correlaciones negativas, estadísticamente significativas, pero de magnitud baja y muy baja, con los factores de inteligencia emocional. Obsesión por las redes sociales (ARS), correlaciona negativamente sólo con Uso de las propias emociones y Regulación de emociones y no correlaciona con Valoración de las emociones propias ni de los demás; Asimismo, Falta de control personal en las redes sociales y Uso excesivo de las RS correlacionan negativamente con Valoración de las emociones propias, Uso de las propias emociones y Regulación de las emociones, con una magnitud baja. Ningún factor del ARS correlaciona con el factor Valoración de las emociones de otros de IE. 
Tabla 1. Correlaciones entre Adicción a Redes Sociales e Inteligencia Emocional

\begin{tabular}{|c|c|c|c|c|c|c|c|}
\hline & Obsesión (RS) & \begin{tabular}{|l|} 
Falta \\
control \\
(RS) \\
\end{tabular} & $\begin{array}{l}\text { Uso } \\
\text { excesivo } \\
\text { (RS) }\end{array}$ & $\begin{array}{l}\text { Valoración } \\
\text { propias } \\
\text { Emoc. }\end{array}$ & $\begin{array}{l}\text { Valorac. } \\
\text { Emoc. } \\
\text { Otros }\end{array}$ & $\begin{array}{l}\text { Uso } \\
\text { propias } \\
\text { Emoc. }\end{array}$ & $\begin{array}{l}\text { Regulación } \\
\text { Emociones }\end{array}$ \\
\hline Obsesión (RS) & - & & & & & & \\
\hline Falta control (RS) & $.58^{* *}$ & - & & & & & \\
\hline Uso excesivo (RS) & $.65^{* *}$ & $.62 * *$ & -- & & & & \\
\hline $\begin{array}{l}\text { Valoración prop. } \\
\text { Emoc. }\end{array}$ & -.10 & $-.17^{* *}$ & $-.14^{*}$ & - & & & \\
\hline $\begin{array}{l}\text { Valorac. Emoc. } \\
\text { Otros }\end{array}$ & .03 & .08 & .03 & $.27^{* *}$ & - & & \\
\hline Uso propias Emoc. & $-.18^{* *}$ & $-.19 * *$ & $-.16 * *$ & $.45^{* *}$ & $.25 * *$ & - & \\
\hline $\begin{array}{l}\text { Regulación } \\
\text { Emociones }\end{array}$ & $-.19 * *$ & $-.22 * *$ & $-.23 * *$ & $.46^{* *}$ & $.13^{*}$ & $.44^{* *}$ & - \\
\hline
\end{tabular}

Adicionalmente, se realizó un análisis del coeficiente de determinación $\left(R^{2}\right)$, considerando a la inteligencia emocional como VI y adicción a redes sociales como VD. Los resultados arrojan puntajes muy bajos de .001 a .09 de determinación, el mayor porcentaje se encuentra entre las dimensiones Obsesión por redes sociales y Valoración de emociones de otros (.09\%).

Se realizó también, un análisis comparativo entre dos grupos que se conformaron de acuerdo a los valores que obtuvieron en la puntuación total del ARS, bajos (0-15) y altos (55-82), se usó la prueba de $U$ de Mann-Whitney y no se hallaron diferencias significativas en las ninguna de las dimensiones de IE, la magnitud del efecto resultó igualmente muy bajo en todos los factores de IE

En la tabla 2, se observan las diferencias de medias en las dimensiones de las dos variables teniendo en cuenta el sexo, éstas oscilan entre -8.86 y 6.63 , lo que determina que, las puntuaciones de varones y mujeres en adicción a redes sociales e inteligencia emocional no presentan diferencias significativas, el tamaño del efecto es considerado entre pequeño ( $d \geq 20$ ) e inexistente; excepto en el factor valoración de las emociones de otros, en el que se aprecia que las mujeres tienen mayores puntajes y una diferencia estadísticamente significativa con respecto a los varones, en el que el tamaño del efecto es también bajo ( $d=.38$ )

Tabla 2. Adicción a Redes Sociales e Inteligencia Emocional según Sexo

\begin{tabular}{|c|c|c|c|c|c|c|}
\hline \multicolumn{2}{|c|}{$\begin{array}{ll} & \text { Grupo }\end{array}$} & \multirow{2}{*}{$\begin{array}{l}\text { Media } \\
10.02 \\
10.10\end{array}$} & \multirow{2}{*}{$\begin{array}{l}U \\
9628\end{array}$} & $p$ & $\Delta$ Media & $d$ \\
\hline Obsesión (RS) & $\begin{array}{l}\text { Fem } \\
\text { Mas }\end{array}$ & & & .89 & -8.86 & -.01 \\
\hline Falta control (RS) & $\begin{array}{l}\text { Fem } \\
\text { Mas }\end{array}$ & $\begin{array}{l}8.65 \\
8.17\end{array}$ & 8771 & .16 & 1.00 & .12 \\
\hline Uso excesivo (RS) & $\begin{array}{l}\text { Fem } \\
\text { Mas }\end{array}$ & $\begin{array}{l}13.38 \\
13.07\end{array}$ & 9373 & .61 & 3.66 & .05 \\
\hline $\begin{array}{l}\text { Valoración } \\
\text { propias Emoc. }\end{array}$ & $\begin{array}{l}\text { Fem } \\
\text { Mas }\end{array}$ & $\begin{array}{l}21.82 \\
22.36\end{array}$ & 8946 & .25 & -1.00 & -.12 \\
\hline $\begin{array}{l}\text { Valoración Emoc. } \\
\text { Otros }\end{array}$ & $\begin{array}{l}\text { Fem } \\
\text { Mas }\end{array}$ & $\begin{array}{l}22.12 \\
20.45\end{array}$ & 7599 & .002 & 2.00 & .38 \\
\hline $\begin{array}{l}\text { Uso propias } \\
\text { Emoc. }\end{array}$ & $\begin{array}{l}\text { Fem } \\
\text { Mas }\end{array}$ & $\begin{array}{l}22.20 \\
22.07\end{array}$ & 9650 & .92 & 6.63 & .03 \\
\hline $\begin{array}{l}\text { Regulación } \\
\text { Emocional }\end{array}$ & $\begin{array}{l}\text { Fem } \\
\text { Mas }\end{array}$ & $\begin{array}{l}19.47 \\
20.46\end{array}$ & 8539 & .08 & -1.00 & -.18 \\
\hline
\end{tabular}


En la tabla 3, se reportan las diferencias de medias de Adicción a redes sociales e inteligencia emocional de acuerdo al tiempo de conexión diario a redes sociales que los propios estudiantes declaran; Se consideró comparar dos grupos: los que tienen tiempos altos de conexión (seis horas o más) y bajos (dos o menos horas). Quienes utilizan seis horas o más de conexión a las RS, tienen puntuaciones más altas en las dimensiones del ARS que aquellos que declaran estar conectados dos horas o menos. La magnitud del efecto de las diferencias de los factores de adicción a redes sociales por el tiempo de conexión a RS, son consideradas de tamaño mediano $(d \geq .50)$ y grandes $(d \geq .80)$. Dentro de ellos, el factor uso excesivo de redes sociales $(d=.91)$ presenta la diferencia más grande. A partir de la comparación realizada, se postula que aquellos que tienen más tiempo de conexión, obtienen mayores puntajes en el ARS que los que tienen menos tiempo de conexión, diferencias con significancia estadística.

Tabla 3. Adicción a Redes Sociales e Inteligencia Emocional según conexión diaria a Redes Sociales

\begin{tabular}{|c|c|c|c|c|c|c|}
\hline & Grupo & Media & $U$ & $p$ & $\Delta$ Media & 0 \\
\hline Obsesión (RS) & $\begin{array}{l}\text { Alta } \\
\text { Baja }\end{array}$ & $\begin{array}{r}11.83 \\
7.14\end{array}$ & 1122 & .001 & 4.00 & .81 \\
\hline Falta control (RS) & $\begin{array}{l}\text { Alta } \\
\text { Baja }\end{array}$ & $\begin{array}{l}9.43 \\
6.83\end{array}$ & 1269 & .001 & 3.00 & .63 \\
\hline Uso excesivo (RS) & $\begin{array}{l}\text { Alta } \\
\text { Baja }\end{array}$ & $\begin{array}{l}15.59 \\
10.24\end{array}$ & 1046 & .001 & 5.00 & .91 \\
\hline Valoración propias Emoc. & $\begin{array}{l}\text { Alta } \\
\text { Baja }\end{array}$ & $\begin{array}{l}22.09 \\
21.71\end{array}$ & 1885 & .61 & 2.86 & .08 \\
\hline Valoración Emoc. Otros & $\begin{array}{l}\text { Alta } \\
\text { Baja }\end{array}$ & $\begin{array}{l}21.71 \\
20.29\end{array}$ & 1608 & .07 & 2.00 & .31 \\
\hline Uso propias Emociones & $\begin{array}{l}\text { Alta } \\
\text { Baja }\end{array}$ & $\begin{array}{l}22.63 \\
22.02\end{array}$ & 1774 & .30 & 1.00 & .12 \\
\hline Regulación Emociones & $\begin{array}{l}\text { Alta } \\
\text { Baja }\end{array}$ & $\begin{array}{l}20.13 \\
21.71\end{array}$ & 1677 & .14 & -1.00 & -.31 \\
\hline
\end{tabular}

Cuando se comparan las diferencias de las medidas de la IE sobre la base de la cantidad de tiempo de conexión a internet, se evidencia que no presentan significancia estadística. Se reportan magnitudes entre pequeños $(d \geq .20)$ e inexistentes.

\section{Discusión}

El planteamiento hipotético de partida contemplaba que se encontrarían correlaciones negativas entre las dimensiones del ARS y las del WLEIS; resultados que eran teóricamente esperados y respaldados por antecedentes que han investigado estas variables (Contreras \& Curo, 2017; Jaramillo, 2018; Sloan et al., 2017); pero lo hallado en este estudio validan sólo parcialmente este supuesto. Se hallaron correlaciones negativas altamente significativas entre Obsesión por las Redes Sociales y Uso de las propias emociones y Regulación de las emociones (Obsesión por las Redes Sociales y Valoración de las propias emociones, no alcanzan la significación estadística, pero la tendencia es negativa); Falta de Control personal en el uso de RS y uso excesivo de las RS, correlacionan con alta significación (aunque con una magnitud baja) con tres factores del WLEIS (valoración de las propias emociones, uso de las propias emociones y regulación de las emociones); De estas relaciones, las más fuertes son los que comprenden la falta de control de RS y regulación de emociones (aunque las magnitudes son bajas), que también han sido reportados por otros autores (Bastidas, 2020; Contreras \& Curo, 2017; Jaramillo, 2018; Marino et al., 2019; Mizera et al., 2019; Sloan et al., 2017; Sola et al., 2013). Por otro lado, la relación negativa del uso excesivo de las RS con regulación emocional, también es respaldada por los hallazgos de Hormes et al. (2014) y Marino et al. (2019); la regulación emocional es un importante factor que afecta a la capacidad para controlar el comportamiento exagerado o exce- 
sivo en general y particularmente el relacionado con el uso de las RS, igualmente puede ayudar en el control de la impulsividad que podría ser un factor importante del uso problemático o del uso excesivo de las RS (Carbonell \& Oberst, 2015; Escurra \& Salas, 2014; Matute, 2016; Turel \& Serenko, 2012).

Estos factores del ARS correlacionan negativamente con la evaluación que las personas realizan de sus emociones, con la forma cómo las utilizan y con la regulación de las mismas. A mayores puntajes en las dimensiones de la escala de adicción a las redes sociales, menores puntuaciones en los factores de la inteligencia emocional o al revés. Lo que lleva a pensar que las altas puntuaciones en el ARS, se relacionan con distorsiones cognitivas y emocionales, que determinan que las personas no poseen una idea clara de lo que ellos mismos buscan o quieren, (Andreassen \& Pallesen, 2014; Granados, et al., 2020); igualmente, con una necesidad de aliviar la tensión y evitar la ansiedad a través de permanecer conectado a ellas por todo el tiempo posible (Salas-Blas, 2019a , 2019b). El adicto al Internet y a las RS se auto engaña y engaña a los demás, minimiza su problema ante el reclamo de los suyos, se propone metas que no logrará, no puede regularse adecuadamente porque más pesa el comportamiento compulsivo (Khoshakhlagh \& Faramarzi, 2012; Andreassen, 2015).

Li et al. (2014) refieren que los individuos que presentan adicción a las redes sociales les cuesta resistirse a la tentación de usar el internet; por ello, es necesario reforzar su autocontrol y autorregulación, cuestión que se encuentra en este estudio (aunque con magnitudes bajas), y que constituyen las principales trabas para el cambio conductual (Caro, 2017; Chóliz \& Marco, 2012; Echeburúa et al., 2014) de los adictos. Este aspecto concreto de los resultados obtenidos puede servir a quienes desarrollan programas terapéuticos o preventivos con adictos a internet o RS, para tener cuidado en planificar el momento oportuno para desarrollar acciones de apoyo social y emocional que requiere una persona comprometida con alguna adicción.

Debido a que las correlaciones halladas eran de magnitudes bajas o pequeñas, se buscó realizar dos análisis adicionales: un análisis de determinación de la IE sobre la ARS, los resultados no encuentran porcentajes de determinación. Igualmente se seleccionó dos grupos de participantes, uno (30 participantes) que obtuvo puntajes totales en el ARS entre 55 y 82 (de un total de 96 posibles) y el otro con una cantidad similar que obtuvo puntuaciones bajas ( 0 a 15); la comparación de las medias no encontró diferencias significativas y la fuerza del efecto son pequeñas, casi inexistentes. Este hallazgo confirma de algún modo los resultados de las correlaciones que son también bajas o muy bajas y pone en el tapete la necesidad de investigar más a fondo las relaciones entre estas dos variables o en general las adicciones comportamentales con variables como la IE, la autoeficacia, la autovaloración, etc. Y pone en duda ideas aceptadas de que estas habilidades podrían constituir una suerte de factores protectores ante las adicciones a las tecnologías y las RS (Echeburúa, 2012; Andreassen \& Pallesen, 2014; Young et al., 2019).

Pero, un resultado que llama la atención es que los tres factores del ARS, no correlacionan con Valoración de las emociones de los demás, se esperaba una correlación negativa como en los otros casos; los datos encontrados dicen que no existe siquiera una tendencia significativa, dan cuenta de una ausencia de relación entre estos factores, que indica que estos son independientes entre sí, es decir, que la conducta del adicto no toma en consideración lo que su entorno piense, diga, o, en general valore. Se ha reflexionado mucho sobre estos datos hallados, se ha descartado que sea el resultado de algún problema de medida. Pero también podría tener sentido si se toma en cuenta que el adicto posee mayor sensibilidad a estímulos relacionados al consumo -sea de sustancias o de su propio comportamiento- y este hecho podría desplazar y restar importancia a las valoraciones que éste le da a estímulos afectivos provenientes de la familia, de los amigos, del entorno en general. Estos individuos están centrados en los estímulos relacionados al reforzamiento negativo (Aguilar et al., 2008; Chicharro et al., 2012; Khoshakhlagh \& Faramarzi, 2012; Salas-Blas, 2019a). Sin embargo, es importante reiterar que este es un resultado difícil de interpretar y que debe ser investigado más a fondo, tal vez con la intervención de factores de la personalidad y con grupos con diagnóstico de uso problemático o de adicción más definidos.

Al comparar la ARS a través del sexo, se halló que hombres y mujeres no se diferencian, sin embargo, existen varios estudios previos que sostienen que los varones usan más internet y las RS (Pomalima, 2016; Salas-Blas, 2019b), pero existen también quienes encontraron lo contrario, las mujeres usan más las RS que los varones (García del Castillo et al. 2019). En cuanto a la IE según sexo, se encontró que las mujeres valoran mejor las emociones de los demás, ellas tienen más interés por las interacciones con los demás y dedican más tiempo que los hombres en cultivar las 
relaciones sociales. Existen estudios que corroboran esta información (Colás et al., 2013; Salas-Blas, 2019a; Salcedo, 2016); es más, algunos autores refieren que el promedio de inteligencia emocional sería significativamente más alto en mujeres (Bueno et al., 2006; Snowden et al., 2015). Sin embargo, otros estudios hallaron resultados diferentes (Shazad \& Bagum, 2012; Wang \& He, 2002). Es posible que los resultados de estos estudios estén ligados a elementos de tipo cultural y del contexto, y, por eso son tan cambiantes.

Se compararon los resultados del cuestionario ARS teniendo en cuenta las horas que pasan conectados a internet (dos ó menos horas y seis ó más horas). Se encontró que quienes tienen más horas de conexión a internet y a redes sociales, tienen también más riesgo de comportarse adictivamente, puesto que no tienen capacidad para controlar su impulsividad para usar las redes, desarrollan alteraciones de tipo cognitivo-afectivo que pueden catalogarse como obsesivos y también desarrollan problemas con su entorno debido al uso excesivo. Esto concuerda con lo encontrado por algunos autores (Mizera et al., 2019; Muñoz-Rivas et al., 2010; Salas \& Escurra, 2014) que reportan una relación directa entre el tiempo de conexión a las redes sociales y la predisposición a ser adictos a ellas. No ha sido posible determinar el nivel de adicción de los grupos comparados por la ausencia de baremos de este instrumento.

Entre las limitaciones que se puede señalar e influir sobre los resultados se encuentran el tipo de participantes de este estudio, son jóvenes que no tienen diagnóstico de adicción a internet ni a RS; otro aspecto limitativo es el uso de un cuestionario construido para medir el nivel de adicción a RS y no otros problemas de uso de las RS; y también, el hecho de administrar los cuestionarios en ambientes de clase y en algunos casos con un profesor presente en el aula, que pudo afectar la deseabilidad social en los jóvenes al considerar que los resultados podrían ser expuestos o evaluados por autoridades de su institución educativa.

Para concluir, Existen correlaciones negativas entre las dimensiones del cuestionario de ARS y de la Inteligencia Emocional, pero lo singular es que la Valoración de las emociones ajenas no se relaciona con ninguna de las dimensiones del ARS; que las mujeres tienden a valorar más las emociones de los demás y que los jóvenes que pasan más tiempo conectados a las redes pueden tener mayor propensión a desarrollar adicción a las redes sociales.

Finalmente, se recomienda investigar más a fondo la relación entre la adicción y la valoración de las emociones de los demás, así como, utilizar muestras más amplias que den cuenta de la magnitud del problema del uso de las RS, en diferentes grupos que componen la población sobre todo de adolescentes y jóvenes.

\section{Referencias}

Aguilar, F., Verdejo, A., López, A., Montañez, M., Gómez, E., Arráez, F., \& Pérez, M. (2008). Respuesta emocional ante estímulos afectivos en sujetos adictos a opiáceos bajo consumo controlado en el PEPSA. Adicciones, 20(1), 27-35. 10.20882/adicciones.286

Andreassen, C. (2015). OnIne Social Network Site Adidiction: A Comprehensive Review. In Current Addiction Reports 2(2), 175-184. https://doi.org/10.1007/s40429-015-0056-9

Andreassen, C., \& Pallesen, S. (2014). Social Network Site Addiction - An Overview. Current Pharmaceutical Design, 20(25), 4053-4061. 10.2174 / 13816128113199990616

Arab, E., \& Díaz, A. (2015). Impacto de las redes sociales e internet en la adolescencia: aspectos positivos y negativos. Revista Médica Clínica Las Condes, 26(1), 7-13. https://doi.org/10.1016/j.rmclc.2014.12.001

Arthanari, S., Khalique, N., Ansari, M., \& Faizi, N. (2017). Prevalence and determinants of internet addiction among indian adolescents. Indian Journal of Community Health, 29(1), 89-95. https://www.iapsmupuk.org/journal/index. php/IJCH/article/view/726/726 
Ato, M., López, J., \& Benavente, A. (2013). Un sistema de clasificación de los diseños de investigación en psicología. Anales de Psicología, 29(3), 1038-1059. https://doi.org/10.6018/analesps.29.3.178511

Bar-On, R. (1997). The Bar-On Emotional Quotient Inventory (EQ-i): A Test of Emotional Intelligence. Toronto: Multi-Health Systems.

Bastidas, L. (2020). Adicción al Internet e Inteligencia Emocional en Adolescentes: Unidad Educativa Juan Pablo II. [Tesis de Pregrado. Universidad Central del Ecuador], Quito, Ecuador.

Bauman, Z. (2011). 44 cartas desde el mundo líquido. Barcelona: Paidós.

Bueno, J., Santana, P., Zerbini, J., \& Ramalho, T. (2006). Inteligência emocional em estudantes universitários. Psicologia, Teoria e Pesquisa, 22(3), 305-316. https://doi.org/10.1590/S0102-37722006000300007

Carbonell, X., \& Oberst, U. (2015). Las redes sociales en línea no son adictivas. Aloma, 33(2), 13-19. https://dialnet. unirioja.es/servlet/articulo?codigo=5267016

Carbonell, X., Chamarro, A., Oberst, U., Rodrigo, B., \& Prades, M. (2018). Problematic Use of the Internet and Smartphones in University Students: 2006-2017. International Journal Environmental Research and Public Health, 15(3), 475. $10.3390 /$ ijerph15030475

Caro, M. (2017). Adicciones tecnológicas: ¿Enfermedad o conducta adaptativa? Medisur, 15(2), 251-260. http:// scielo.sld.cu/scielo.php?script=sci_arttext\&pid=S1727-897X2017000200014

Cía, A. (2017) Las adicciones no relacionadas a sustancias (DSM-5, APA, 2013): Un primer paso para la inclusión de las adicciones conductuales en las clasificaciones categoriales vigentes. Revista del hospital Interzonal General de Agudos 6(1), 32-37. 10.20453/rnp.2013.1169

Chicharro, J., Pérez-García. J., \& Sanjuán. P. (2012). Respuesta emocional en adictos a sustancias en tratamiento ambulatorio. Adicciones, 24(1), 59-68. https://doi.org L10 20882 Ladicciones. 118

Chóliz, M., \& Marco, C. (2012). Adicción a Internet y redes sociales. Tratamiento psicológico. Madrid: Alianza Editorial.

Clement, J. (9 de agosto de 2020). Población digital mundial a Julio de 2020. https://www.statista.com/statistics/278414/number-of-worldwide-social-network-users/

Colás, P., González, T., \& de Pablos, J. (2013). Young people and social networks: Motivations and preferred uses. Comunicar, 20(40), 15-23. https://doi.org/10.3916/C40-2013-02-01

Contreras, P., \& Curo, M. (2017). Dependencia a las redes sociales e inteligencia emocional en estudiantes de una Universidad Privada de Chiclayo 2017. [Tesis de pregrado. Universidad Señor de Sipán], Pimentel, Perú.

Del Cid, A., Méndez, R., \& Sandoval, F. (2011). Investigación. Fundamentos y metodología. 2a ed. México: Prentice Hall

DeVellis, R. (2011). Scale Development, Theory and Applications. 3a ed. Los Ángeles: SAGE. Echeburúa, E. (2016). Abuso de Internet: ¿antesala para la adicción al juego de azar online? Madrid: Pirámide. 
Echeburúa, E. (2012). Factores de riesgo y factores de protección en la adicción a las nuevas tecnologías y redes sociales en jóvenes y adolescentes. Revista Española de Drogodependencia, 37(4), 435-447. https://dialnet.unirioja. es/servlet/articulo?codigo $=4113810$

Echeburúa, E. (2016). Abuso de Internet: ¿̇antesala para la adicción al juego de azar online? Madrid: Pirámide.

Echeburúa, E., \& De Corral, P. (2010). Adicción a las nuevas tecnologías y a las redes sociales en jóvenes: un nuevo reto. Adicciones, 22(2), 91-96. https://doi.org/10.20882/adicciones.196

Echeburúa, E., \& Requesens, A. (2012). Adicción a las redes sociales y nuevas tecnologías en niños y adolescentes. Madrid: Pirámide.

Echeburúa, E., Salaberría, K., \& Cruz, M. (2014). Nuevos retos en el Tratamiento de Juego Patológico. Terapia psicológica 32(1), 31-40. http://dx.doi.org/10.4067/\$0718-48082014000100003

Escurra, M., \& Salas, E. (2014). Construcción y validación del cuestionario de Adicción a Redes Sociales (ARS). Liberabit. Revista de Psicología, 20(1), 73-91. https://biblat.unam.mx/es/revista/liberabit/articulo/construccion-y-validacion-del-cuestionario-de-adiccion-a-redes-sociales-ars

Faul, F., Erdfelder, E., Lang, A., \& Buchner, A. (2007). G*Power 3: A flexible statistical power analysis program for the social, behavioral, and biomedical sciences. Behavior Research Methods, 39(2), 175-191. https://doi.org/10.3758/ BF03193146

Fuster, H., Chamarro, A., \& Oberst, U. (2017). Fear of Missing Out, online social networking and mobile phone addiction: A latent profile approach. Aloma, 35(1), 23-30. https://www.researchgate.net/publication/317167392 Fear of Missing_Out online social networking_and mobile_phone addiction_A latent_profile approach

García del Castillo, J., García del Castillo-López, A., Dias, P., \& García-Castillo, F. (2019). Conceptualización del comportamiento emocional y la adicción a las redes sociales. Health and Addictions, 19(2), 173-181. http://dx.doi. org/10.21134/haaj.v19i2.525

Goleman, D. (1995). Inteligencia Emocional. Barcelona: Kairós.

Goleman, D. (2011). Working With Emotional Intelligence. New York: Bantam Books.

Gómez, P., Rial, A., Braña, T., Golpe, S., \& Varela, J. (2017). Screening of Problematic Internet Use among Spanish Adolescents: Prevalence and Related Variables. Cyberpsychology, Behavior, and Social Networking, 20(4), $259-267$. https://doi.org/10.1089/cyber.2016.0262

Granados, B., Quintana-Orts, C., \& Rey, L. (2020). Regulación emocional y uso problemático de las redes sociales en adolescentes: el papel de la sintomatología depresiva. Healths and Adicttions, 20(1), 77-86. https://doi. org/10.21134/haaj.v20i1.473

Hormes, J., Kearns, B., \& Timko, C. (2014). Craving Facebook? Behavioral addiction to online social networking and its association with emotion regulation deficits. Addiction, 109(12), 2079-2088. 10.1111 / add.12713

Hütt, H. (2012). Las redes sociales. Una nueva herramienta de difusión. Reflexiones, 91(2). 121-128. 10.15517/RR. $\underline{\text { V91I2.1513 }}$ 
Instituto Nacional de Estadística e Informática. (2020). Las Tecnologías de Información y Comunicación en los Hogares: Octubre-Noviembre-Diciembre 2019. https://www.inei.gob.pe/biblioteca-virtual/boletines/tecnologias-de-la-infor$\underline{\text { maciontic/1/ }}$

IPSOS (2018). La generación Z. https://www.ipsos.com/es-es/la-generacion-z

Jaramillo, M. (2018). Relación entre inteligencia emocional con indicadores de adicción a las redes sociales en estudiantes de un instituto público de Tingo maría 2017. [Tesis de maestría. Universidad Peruana Cayetano Heredia], Lima, Perú.

Katz, M. (2011). Multivariable analysis: A practical guide for clinicians and Public Health Reserchers. 3a ed. New York: Cambridge University Press.

Kawabe, K., Horiuchi, F., Ochi, M., Oka, Y., \& Ueno, S. (2016). Internet addiction: Prevalence and relation with mental states in adolescents. Psychiatry and Clinical Neurosciences, 70(9), 405-412. 10.1111/ pcn.12402

Khoshakhlagh, H., \& Faramarzi, S. (2012). The Relationship of Emotional Intelligence and Mental Disorders with Internet Addiction in Internet Users University Students. Addiction \& Health, 4(3-4), 133-141. https://www.ncbi.nlm. nih.gov/pmc/articles/PMC3905545/

Law, K., Wong, C., \& Song, L. (2004). The construct and criterion validity of emotional intelligence and its potential utility for management studies. In Journal of Applied Psychology, 89(3), 483-496. https://doi.org/10.1037/0021$\underline{9010.89 .3 .483}$

Leung, L. (2007). Stressful life events, motives for internet use, and social support among digital kids. Cyberpsychology and Behavior, 10(2), 204-214. https://doi.org/10.1089/cpb.2006.9967

Li, C., Dang, J., Zhang, X., Zhang, Q., \& Guo, J. (2014). Internet addiction among Chinese adolescents: The effect of parental behavior and self-control. Computers in Human Behavior, 41(1), 1-7. https://doi.org/10.1016/j. chb.2014.09.001

Marino, C., Caselli, G., Lenzi, M., Monaci, G., Vieno, A., Nikčević, V., \& Spada, M. (2019). Emotion Regulation and Desire Thinking as Predictors of Problematic Facebook Use. Psychiatric Quarterly, 90(2), 405-411. https://doi. org/10.1007/s11126-019-09628-1

Matalinares, M., Arenas, C., Díaz, G., \& Dioses, A. (2013). Adicción a la internet y agresividad en estudiantes de secundaria del Perú. Revista de investigación en psicología, 16(1), 75-93. https://doi.org/10.15381/rinvp.v16i1.3920

Matassi, M., \& Boczkowski, P. (2020). Redes sociales en Iberoamérica. Artículo de revisión. El profesional de la información, 29(1), 1-21. https://doi.org/10.3145/epi.2020.ene.04

Matute, H. (2016). ¿Adicción, abuso o uso problemático de Internet?. En Abuso de Internet: ¿antesala para la adicción al juego de azar online? (pp. 31-50). Madrid: Pirámide.

Merino, C., Lunahuaná-Rosales, M., \& Pradhan, K. (2016). Validación estructural del Wong-Law Emotional Intelligence Scale (WLEIS): estudio preliminar en adultos. Liberabit: Revista Peruana de Psicología, 22(1), 103-110. https://doi. org/10.24265/liberabit.2016.v22n1.09

Mizera, S., Jastrzębska, K., Cyganek, T., Bąk, A., Michna, M., Stelmach, A.,... Janas-Kozik, M. (2019). The Relation 
Between Emotional Intelligence and Internet Addiction in Katowice High School Students. Psychiatria Danubina, 31(3), 568-573. https://www.researchgate.net/publication/336776740 The Relation Between Emotional Intelligence and Internet_Addiction in Katowice_High_School_Students

Muñoz-Rivas, M., Fernández, L., \& Gámez-Guadix, M. (2010). Analysis of the indicators of pathological internet use in spanish university students. The Spanish Journal of Psychology, 13(2), 697-707. https://doi.org/10.1017/ $\underline{\text { S1138741600002365 }}$

Peña, B., \& Rubio, M. (2013). Gestión de la emoción en la comunicación mediada por ordenador (CMO). Estudios Sobre El Mensaje Periodístico, 19(0), 905-913. https://doi.org/10.5209/rev ESMP.2013.v19.42174

Perrella, R., \& Caviglia, G. (2017). Internet addiction, self-esteem, and relational patterns in adolescents. Clinical Neuropsychiatry, 14(1), 82-87. https://www.researchgate.net/publication/316597717 Internet addiction self-esteem and relational patterns in adolescents

Plaza, J. (2016). Impacto de las redes sociales virtuales en estudiantes adolescentes: Informe de investigación / Virtual Social nets Impact on Teenage Students: a Research Report. Revista Internacional de Tecnologías En La Educación, 3(1), 53-63. https://doi.org/10.37467/gka-revedutech.v3.281

Pomalima, R., Palpán, J., Caballero, J. \& Cruz, V._(2016). Prevalencia de Riesgo de la Conducta Adictiva a Internet entre los adolescentes de Lima Metropolitana y Arequipa. Informe final. Fundación MAPFRE. https://www.mapfre. com.pe/media/Resumen-del-Estudio-Prevalencia-de-riesgo-de-la-conducta-adictiva-a-internet-Web.pdf

Pontes, H., Taylor, M., \& Stavropoulos, V. (2018). Beyond "facebook Addiction": The Role of Cognitive-Related Factors and Psychiatric Distress in Social Networking Site Addiction. Cyberpsychology, Behavior, and Social Networking, 21(4), 240-247. https://doi.org/10.1089/cyber.2017.0609

Salas, E. (2014). Adicciones psicológicas y los nuevos problemas de salud. Cultura, 28, 111-146. http://www.revistacultura.com.pe/revistas/RCU_28_1_adicciones-psicologicas-y-los-nuevos-problemas-de-salud.pdf

Salas, E. \& Escurra, M. (2014). Uso de redes sociales entre estudiantes limeños. Revista Peruana de Psicología y Trabajo Social, 3(1), 75-90. http://revistas.uigv.edu.pe/index.php/psicologia/article/view/57

Salas-Blas, E. (2019a). Las adicciones comportamentales. En C. Rojas-Jara (Ed.), Drogas: Sujeto, Sociedad y Cultura (pp. 123-138). Talca: Nueva Mirada Ediciones.

Salas-Blas, E. (2019b). Patrones de uso y abuso de las TIC entre adolescentes de Lima y Arequipa. Percepción de los riesgos. Informe final. Fundación MAPFRE. https://app.mapfre.com/documentacion/publico/es/catalogo_imagenes/grupo.do?path=1102847

Salas-Blas, E., Copez-Lonzoy, A., \& Merino-Soto, C. (2020). ¿Realmente es demasiado corto?. Versión breve del cuestionario de Adicción a Redes Sociales (ARS-\&). Health and Addictions, 20(2), 105-118. http://dx.doi.org/10.21134/ haaj.v20i2.536

Salcedo, I. (2016). Relación entre adicción a redes sociales y autoestima en jóvenes universitarios de una universidad particular de Lima. [Tesis de maestría. Universidad San Martin de Porres], Lima. https://alicia.concytec.gob.pe/ vufind/Record/USMP 07cb8c7de1a52b95706b7965c4c1327b

Salovey, P., \& Mayer, J. (1990). Emotional Intelligence. Imagination, Cognition and Personality, 9(3), 185-211. https:// doi.org/10.2190/DUGG-P24E-52WK-6CDG 
Saraiva, J., Esgalhado, G., Pereira, H., Monteiro, S., Afonso, R., \& Loureiro, M. (2018). The relationship between emotional intelligence and internet addiction among youth and adults. Journal of Addictions Nursing, 29(1), 13-22. 10.1097 / JAN.0000000000000209

Shazad, S., \& Bagum, N. (2012). Gender differences in trait emotional intelligence: A comparative study. Business Review, 7(2), 106-112. https://bit.ly/3aceK8L

Sloan, E., Hall, K., Moulding, R., Bryce, S., Mildred, H., \& Staiger, P. (2017). Emotion regulation as a transdiagnostic treatment construct across anxiety, depression, substance, eating and borderline personality disorder: a systematic review. Clinical Psychology Review, 57(1), 141- 163. https://doi.org/10.1016/i.cpr.2017.09.002

Snowden, A., Stenhouse, R., Young, J., Carver, H., Carver, F., \& Brown, N. (2015). The relationship between emotional intelligence, previous caring experience and mindfulness in student nurses and midwives: A cross sectional analysis. Nurse Education Today, 35(1), 152-158. https://doi.org/10.1016/i.nedt.2014.09.004

Sola, J., Rubio, G., \& Rodríguez, F. (2013). La impulsividad: ¿Antesala de las adicciones comportamentales?. Revista Salud y Drogas, 13(2), 145-155. http://dx.doi.org/10.21134/haaj.v13i2.211

The jamovi project. (2019). Jamovi. (Version 1.0) [Computer Software]. https://www.jamovi.org

Turel, O., \& Serenko, A. (2012). The benefits and dangers of enjoyment with social networking websites. European Journal of Information Systems, 21(5), 512-528. https://doi.org/10.1057/ejis.2012.1

Ventura-León, J., \& Caycho. T. (2017). El coeficiente Omega: un método alternativo para la estimación de la confiabilidad. Revista Latinoamérica de Ciencias Sociales, Niñez y Juventud, 15(1), 625-627. https://www.researchgate.net/ publication/313623697 El coeficiente_Omega un metodo_alternativo_para la estimacion de la confiabilidad

Wang, C., \& He. Z. (2002). The Relationship between Parental Rearing Styles with General Self-efficacy and Emotional Intelligence in Middle School students. Chinese Mental Health Journal, 16(11), 781-785. http://en.cnki.com.cn/ Article en/CJFDTOTAL-ZXWS200211022.htm

Watters, C., Keefer, K., Kloosterman, P., Summerfeldt, L., \& Parker, J. (2013). Examining the structure of the Internet Addiction Test in adolescents: A bifactor approach. Computers in Human Behavior, 29(6), 2294-2302. https://doi. org/10.1016/i.chb.2013.05.020

Wong, C., \& Law, K. (2002). The effects of leader and follower emotional intelligence on performance and attitude: An exploratory study. Leadership Quarterly, 13(3), 243-274. https://doi.org/10.1016/S1048-9843(02)00099-1

Young, K., Sandman, C., \& Craske, M. (2019). Positive and negative emotion regulation in adolescence: links to anxiety and depression. Brain Sciences, 9(4), 76-94. https://doi.org/10.3390/brainsci9040076

Yu, L., \& Shek, D. (2013). Internet addiction in Hong Kong adolescents: A three-year longitudinal study. Journal of Pediatric and Adolescent Gynecology, 26(3), 10-17. https://doi.org/10.1016/i.jpag.2013.03.010 